\title{
Use of Tensor Formats in Elliptic Eigenvalue Problems
}

\author{
Wolfgang Hackbusch, Boris N. Khoromskij* \\ Max-Planck-Institut für Mathematik in den Naturwissenschaften, \\ Inselstr. 22-26, D-04103 Leipzig, Germany; \{wh, bokh\}@mis.mpg.de \\ Stefan Sauter \\ Universität Zürich, Switzerland; stas@math.uzh.ch \\ Eugene E. Tyrtyshnikov ${ }^{\dagger}$ \\ Institute of Numerical Mathematics, Russian Academy of Sciences, \\ Gubkina 8, 119333 Moscow, Russia; tee@inm.ras.ru
}

\begin{abstract}
We investigate approximations by finite sums of products of functions with separated variables to eigenfunctions of certain class of elliptic operators in higher dimensions, and especially conditions providing an exponential decrease of the error with respect to the number of terms. The results of the consistent use of tensor formats can be regarded as a base for a new class of rank truncated iterative eigensolvers with almost linear complexity in the univariate problem size that improves dramatically the traditional methods of linear scaling in the volume size. Tensor methods can be applied to solving large scale spectral problems in the computational quantum chemistry, for example to the Schrödinger, Hartree-Fock and Kohn-Sham equations in electronic structure calculations. The results of numerical experiments clearly indicate the linear-logarithmic scaling of low-rank tensor method in the univariate problem size. The algorithms work equally well for the computation of both, minimal and maximal eigenvalues of the discrete elliptic operators.
\end{abstract}

AMS Subject Classification: 65F30,65F50,65N35, 65F10

Key words: elliptic operators, spectra, eigenfunctions, separation of variables, separable approximations, matrix approximations, low-rank matrices, Kronecker products, multi-dimensional matrices, tensors, numerical methods, iterative algorithms

\footnotetext{
${ }^{*}$ The work of the second author was partially supported by FIM of Switzerland during his visit of the ETH/University of Zürich in May - July, 2008.

†This work was partially supported by RFBR/DFG grant 09-01-91332, RFBR grants 08-01-00115, 09-0112058 and the Priority Research Grant of the Department of Mathematical Sciences of Russian Academy of Sciences.
} 


\section{Introduction}

Recent advances in tensor approximation methods applied to the functions and operators in $\mathbb{R}^{d}$ lead to the natural idea of solving multi-dimensional boundary and eigenvalue problems in tensor-product formats. This idea has been first time formulated in [3] in the very general setting. The recent results on efficient methods of separable tensor approximation of multivariate functions and their application in electronic structure calculations can be found in $[12,4,6,9,16,18,19]$ and others. The concept of separation of variables, that is the key idea of tensor-product numerical approximation, allows to get rid of the "curse of dimensionality" inherent to the traditional numerical methods of linear complexity in the volume, $O\left(n^{d}\right)$, where $n$ is the one-dimensional grid size. The goal of tensor-structured numerical methods is thus the construction of numerical algorithms in high dimension that scale linearly in both $d \gg 1$ and $n, O(d n)$. Notice that tensor methods are applicable to the discretisations on tensor-product grids, which is often the case in multidimensional setting.

In this paper we investigate approximations by finite sums of products of functions with separated variables to eigenfunctions of elliptic operators with smooth coefficients, and especially conditions providing an exponential decrease of error in the number of terms. We demonstrate that tensor-truncated version of the simple inverse power iteration allows to compute few minimal eigenvalues of the discrete elliptic operator with the complexity $O(n)$, on large $n \times n \times \ldots \times n$ tensor grid in $\mathbb{R}^{d}$. Tensor-truncated iteration for solving the spectral problems can be inplemented in the efficient way based on the already existing methods of separable approximation of multivariate functions and operators $[13,12,26,15,16,5,17]$.

We consider the model eigenvalue problem: Find a pair $(\lambda, u) \in \mathbb{C} \times H_{0}^{1}(\Omega) \backslash\{0\}$ such that

$$
\begin{aligned}
& \Lambda u=\lambda u \text { in } \Omega \text {, } \\
& u=0 \quad \text { on } \partial \Omega
\end{aligned}
$$

with the elliptic differential operator $\Lambda$ of the form

$$
\Lambda u:=-\operatorname{div}(A \operatorname{grad} u)+\langle b, \nabla u\rangle+c u,
$$

where $\Omega \in \mathbb{R}^{d}$ is some bounded or unbounded rectangular-type domain (in the latter case, we assume the exponential decay of the soltion $u(x)$, as $x \rightarrow \infty)$, and the operator coefficients $A, b, c$ in ( $1.1 \mathrm{~b}$ ) are supposed to be smooth (analytic) in $\Omega$.

We will prove that the eigenfunctions of problem ( $1.1 \mathrm{a})$ allow separable approximation that converges exponentially in the number of terms.

Problem ( $1.1 \mathrm{a}$ ) is discretised by the Galerkin FEM with tensor-product basis functions, so that the arising stiffness and mass matrices of size $n^{\otimes d}$ (with $d$-fold product $n^{\otimes d}=$ $n \times \ldots \times n)$ are represented in the low Kronecker rank format with the storage requirements and computational complexity of order $O(d n)$.

In this paper, our focus is to break the curse of dimension in numerical solution of high-dimensional elliptic eigenvalue problems. Hence, for the ease of presentation, we use simple iterative solvers such as the power method or the inverse power iteration, though algorithms of better choice can be easily adapted to our concept. Due to the above mentioned approximation results for the continuous solutions, and relying on the rank-structured representation of all matrices involved, we propose to solve the corresponding high-dimensional algebraic eigenvalue problem of the size $n^{\otimes d}$ in the low tensor-rank format. To this end, 
we introduce the so-called "truncated iterations", where most of the intermediate vectors have to be approximated in some fixed rank-structured tensor product form with the storage size $O(n)$. The corresponding rank truncation performed at each iteration can be based on recently developed methods (cf. [18, 8, 24]). For the class of rank structured matrices, our algorithm can be shown to have storage and complexity bounds of order $O\left(r^{d} n+r n d\right)$, or even $O(d R r n)$, where $r, R$ are the small (often fixed) rank parameters with the theoretical bounds $r=O(\log n)$ and $R=O(\log n|\log \varepsilon|)$.

We summarise that the main concept of tensor-structured solution methods includes the following ingredients

- Existence of the low separation rank approximation to the exact solution in $\mathbb{R}^{d}$.

- Construction of the fix-point type iteration that allows the rank truncation procedure at each iterative step.

- Representation of all matrices approximating $\Lambda$ in the low Kronecker rank format.

- Using the efficient rank reduction algorithms for higher order tensors.

The results of the consistent use of tensor formats can be regarded as a base for a new class of iterative eigensolvers in higher dimensions, providing almost linear complexity in the univariate problem size, $O(n)$. Such algorithms can be applied, in particular, in numerical linear algebra, quantum chemistry, as well as in the stochastic PDEs for elliptic spectral problems.

We notice that in the case $d=2$ the rank truncation operator is realised by the "truncated SVD" method applied to the rank- $R, n \times n$ matrix. It is, in practice, a finite algorithm (with complexity at most $\left.O\left(n R^{2}+R^{3}\right)\right)$ providing the best rank-r approximation to the current iterand (see numerics in Sections 2 and 6.2). Hence, numerical results for $d=2$ can be viewed as the reference cases, demonstrating nearly optimal performance of the proposed techniques.

The rest of the paper is organized as follows. In Section 2 we give motivating illustrations for the Laplace operator, and for the simple Schrödinger equation in the case of Hydrogen atom, which clearly indicate the approximability of the solutions by few separable functions. In Section 3, we briefly describe the tensor product formats for representing multivariate functions of the continuous and discrete arguments. Section 4 discusses the diecretization of elliptic eigenvalue problems in tensor-structured formats and introduces the iterative solvers with rank truncation. Section 5 proves the existence of separable approximation for the eigenfunctions of certain class of elliptic operators posed in $\mathbb{R}^{d}$. This result is of principal significance for understanding the rigorous mathematical basis for applying tensor methods in multidimensional setting. Section 6 presents numerical illustrations on the efficiency of tensor formats for certain spectral problems in dimensions $d=2,3$. 


\section{Simple Motivating Examples}

We begin with simple example just to demonstrate the basis idea of the tensor method. In particular, we are motivated by the nice solution structure for the 2D Laplace operator

$$
\Delta=\frac{\partial^{2}}{\partial x^{2}}+\frac{\partial^{2}}{\partial y^{2}}
$$

and the eigenproblem

$$
-\Delta u(x, y)=\lambda u(x, y), \quad(x, y) \in[0, \pi]^{2} .
$$

If $u$ is zero on the boundary, then the eigenvalues and eigenfunctions are

$$
\lambda_{k l}=k^{2}+l^{2}, \quad u_{k l}(x, y)=\sin k x \sin l y, \quad k, l=1,2, \ldots
$$

A matrix counterpart of the Laplace operator can be taken in the form

$$
M u=\lambda u, \quad M=A \otimes I+I \otimes A,
$$

where $\otimes$ denotes the Kronecker (tensor) product, $I$ is the identity and

$$
A=\left[\begin{array}{rrrrr}
2 & -1 & & & \\
-1 & 2 & -1 & & \\
& \cdots & \ldots & \ldots & \\
& & -1 & 2 & -1 \\
& & & -1 & 2
\end{array}\right]
$$

Let $A$ and $I$ be of order $n$. Then solution of the matrix eigenvalue problem $M x=\lambda x$ can be represented in the explicit form. The eigenvalues are

$$
\lambda_{k l}=4 \sin ^{2} \frac{\pi k}{2(n+1)}+4 \sin ^{2} \frac{\pi l}{2(n+1)}, \quad 1 \leq k, l \leq n,
$$

and the corresponding eigenvectors are exactly represented as tensor products:

$$
x_{k l}=u^{k} \otimes v^{l},
$$

where $u^{k}$ and $v^{l}$ are $n$-dimensional vectors with the entries

$$
u_{s}^{k}=\sin \frac{\pi k s}{n+1}, \quad 1 \leq s \leq n ; \quad v_{t}^{l}=\sin \frac{\pi l t}{n+1}, \quad 1 \leq t \leq n .
$$

In general, the cost of traditional iterative eigenvalue algorithms is higher than linear, possibly $O\left(n^{2}\right)$. The tensor structure of eigenvectors allows to modify the eigensolvers so that the cost of one iteration reduces to $O(n)$. The above argument remains valid also for $d$-Laplacian defined on $[0, \pi]^{d}$. Numerical examples on the efficiency of iterative eigensolvers in the case of well separable solution will be given in Section 6 . 
The second example shows the typical situation when the well separable solution can be found only approximately. We consider the solution of the Schrödinger equation for hydrogen atom,

$$
\left(-\frac{1}{2} \Delta-\frac{1}{\|x\|}\right) u=\lambda u, \quad u \in H_{0}^{1}\left(\mathbb{R}^{3}\right)
$$

given by $u(x)=e^{-\|x\|}$ and for $\lambda=-0.5$. It is proven to have the low-rank separable approximation on the continuous and the discrete levels $[12,16]$. In particular, there exist constants $c_{k} \in \mathbb{R}$ and $\lambda_{k} \in \mathbb{R}_{>0}$, such that

$$
\left|e^{-\|x\|}-\sum_{k=1}^{M} c_{k} e^{-\lambda_{k}\|x\|^{2}}\right| \leq C e^{-\alpha \sqrt{M}}, \quad \alpha>0, \quad x \in[-\beta, \beta]^{3},
$$

which means that the accuracy $\varepsilon>0$ can be achieved with the number of terms $M=$ $\left(|\log \varepsilon|^{2}\right)$ (exponentially fast convergence). Similar rank- $M$ approximation can be derived for the Newton potential $\frac{1}{\|x\|}$. Moreover, in view of the exponential decay in the solution as $\|x\| \rightarrow \infty$, we can formulate the "approximating" problem in the finite hypercube imposing homogeneous Dirichlet conditions at the boundary.

Thus, we are interested to find tensor formats in matrix eigensolvers for more general elliptic problems,

$$
\Lambda u=\lambda u, \quad u:[0, a]^{d} \rightarrow \mathbb{R},
$$

where $\Lambda$ is the elliptic operator with smooth enough (or separable) coefficients. For this purpose, we next introduce the tensor representations.

\section{Description of tensor formats}

\subsection{Tensor spaces and tensor representations}

Several continuous and discrete spaces considered in this paper are tensor spaces of order $d$, where in our application $d$ equals the spatial dimension of the eigenvalue problem. Let

$$
\mathbb{W}=W_{1} \otimes W_{2} \otimes \ldots \otimes W_{d}
$$

be the notation for the underlying tensor space. (Note that in the applications we have in mind, $\mathbb{W}$ is some Sobolev space, e.g., $H_{0}^{1}(\Omega)$ for some product domain $\Omega=\Pi_{j=1}^{d}\left(a_{j}, b_{j}\right)$ and $W_{j}=H_{0}^{1}\left(\left(a_{j}, b_{j}\right)\right)$.)

In this subsection, we will explain the form in which we will seek the approximate eigenvector.

By definition, each $w \in \mathbb{W}$ can be written as a sum

$$
w=\sum_{k} w_{k}^{(1)} \otimes w_{k}^{(2)} \otimes \ldots \otimes w_{k}^{(d)} \quad\left(w_{k}^{(j)} \in W_{j}\right) .
$$

The first representation, usually called the canonical format, is given by defining the subset of those elements in $\mathbb{W}$ which require only $R$ terms for their representation. They form the set

$$
\mathcal{C}_{R}=\left\{w \in \mathbb{W}: w=\sum_{k=1}^{R} w_{k}^{(1)} \otimes w_{k}^{(2)} \otimes \ldots \otimes w_{k}^{(d)}, w_{k}^{(j)} \in W_{j}\right\} .
$$


We say that elements $w \in \mathcal{C}_{R}$ with $w \notin \mathcal{C}_{R-1}$ have the tensor rank $R$. Obviously, tensors $w \in \mathcal{C}_{R}$ can be represented by the description of the $R d$ elements $w_{k}^{(j)} \in W_{j}$. Hence, the cost for their representation scales linear in $d$.

Next, we will introduce the Tucker representation. For given Tucker rank $\mathbf{r}=\left(r_{j}\right)_{j=1}^{d} \in$ $\mathbb{N}^{d}$ we set

$$
\mathcal{M}_{\mathbf{r}}:=\left\{v \in \mathbb{W} \mid \forall 1 \leq j \leq d \exists V_{j} \text { subspace of } W_{j} \text { with } \operatorname{dim} V_{j}=r_{j} \text { and } v \in \mathbb{V}=\bigotimes_{i=1}^{d} V_{j}\right\}
$$

Then, a representation of $w \in \mathcal{M}_{\mathbf{r}}$ in the Tucker format (3.4) is given by

$$
v=\sum_{\mathbf{k}} b_{\mathbf{k}} \phi_{k_{1}}^{(1)} \otimes \phi_{k_{2}}^{(2)} \otimes \ldots \otimes \phi_{k_{d}}^{(d)}
$$

where the multi-index $\mathbf{k}=\left(k_{1}, \ldots, k_{d}\right)$ runs over all $1 \leq k_{j} \leq r_{j}, 1 \leq j \leq d$, and $\left(\phi_{k}^{(j)}\right)_{k=1}^{r_{j}}$ denotes some basis of $V_{j}$ appearing in definition of $\mathcal{M}_{\mathbf{r}}$ (cf. (3.3)). This representation requires $\prod_{j=1}^{d} r_{j}$ real numbers and the storage of the $\sum_{j=1}^{d} r_{j}$ vectors $\phi_{k}^{(j)}$.

\subsection{Tensor approximation, tensor truncation}

For any element $w \in \mathbb{W}$ there are numbers $R$ and tuples $\mathbf{r}$ such that $w \in \mathcal{C}_{R^{*}}$ and $w \in \mathcal{M}_{\mathbf{r}^{*}}$, but the ranks $R^{*}$ and $\mathbf{r}^{*}$ may be rather large. A representation by one of the tensor formats (3.2) or (3.4) is only of interest if the respective ranks are small enough. Therefore, given $w \in \mathbb{W}$ we search for approximations $v \in \mathcal{C}_{R}$ or $v \in \mathcal{M}_{\mathbf{r}}$ with suitably small ranks $R$ or $\mathbf{r}$. When we fix the set $\mathcal{S}:=\mathcal{C}_{R}$ or $\mathcal{S}:=\mathcal{M}_{\mathrm{r}}$, the smallest error is described by

$$
\sigma(w, \mathcal{S}):=\inf _{v \in \mathcal{S}}\|w-v\|
$$

In the following we will give examples where $\sigma(w, \mathcal{S})$ decays exponentially with the rank $R$ or $\min \left\{r_{j}: 1 \leq j \leq d\right\}$, respectively. In general, the infimum in the definition of $\sigma(w, \mathcal{S})$ cannot be replaced by a minimum, since for $\mathcal{S}=\mathcal{C}_{R}$ a minimiser is not necessarily existing. Although, the minimiser exists for $\mathcal{S}=\mathcal{M}_{\mathbf{r}}$, its computation can be performed only approximately. Therefore, in practice, one has to determine a $v \in \mathcal{S}$ such that $\|w-v\|$ comes close to $\sigma(w, \mathcal{S})$. The replacement of $w$ by such a $v \in \mathcal{S}$ is called the tensor truncation to $\mathcal{S}$ and denoted by

$$
w \mapsto v= \begin{cases}T_{R} w & \text { if } \mathcal{S}=\mathcal{C}_{R} \\ T_{\mathbf{r}} w & \text { if } \mathcal{S}=\mathcal{M}_{\mathbf{r}}\end{cases}
$$

Heuristic methods for computing the rank structured approximations in different problem settings are discussed in $[7,21,30,18,9,8]$.

In the particular case of $d=2$, the difficulties mentioned above do not appear. The minimiser of $\inf _{v \in \mathcal{S}}\|w-v\|=\min _{v \in \mathcal{S}}\|w-v\|$ is the result of the truncated singular value decomposition. Furthermore, the representations by $\mathcal{C}_{R}$ and $\mathcal{M}_{\mathbf{r}}$ with $\mathbf{r}=(R, R)$ coincide. 


\subsection{Application to function spaces}

Let $I=I_{1} \times I_{2} \times \ldots \times I_{d}$ be the product of (possibly infinite) intervals $I_{j} \subset \mathbb{R}$. Then $L^{2}(I)$ is the tensor space $L^{2}\left(I_{1}\right) \otimes L^{2}\left(I_{2}\right) \otimes \ldots \otimes L^{2}\left(I_{d}\right)$. The tensor product $w=\bigotimes_{j=1}^{d} w^{(j)}$ of $w^{(j)} \in L^{2}\left(I_{j}\right)$ corresponds to the pointwise product $w(x)=\prod_{j=1}^{d} w^{(j)}\left(x_{j}\right)$.

If $w$ is an analytical function in all variables $x_{j}$, approximations by polynomials may lead to small errors. In the case of a uniform polynomial degree $r-1$, the subspaces $V_{j} \subset W_{j}$ in (3.3) are $\mathbb{P}_{r-1}$ and any $v \in \mathbb{V}=\bigotimes_{j=1}^{d} V_{j}$ has the Tucker rank $\mathbf{r}=(r, \ldots, r)$. The error, which is an upper bound of $\sigma\left(w, \mathcal{M}_{\mathbf{r}}\right)$, depends on the decay of the higher derivatives. The analysis in Section 5 will show exponential decay of $\sigma\left(w, \mathcal{M}_{\mathbf{r}}\right)$.

Multivariate functions depending on the Euclidean norm as, e.g., the classical potentials $1 /\|x\|, e^{-\lambda\|x\|} /\|x\|, e^{-\lambda\|x\|}$ can be rather well approximated in $\mathcal{C}_{R}$ leading to exponential decay of $\sigma\left(w, \mathcal{C}_{R}\right)$ with respect to $R$. For its computation and analysis see $[13,12,26,15,16,5]$.

\subsection{Application to grid functions}

Galerkin discretisations with tensor product basis functions or finite difference schemes lead to grid points $x_{\mathbf{i}}=\left(x_{i_{1}}^{(1)}, \ldots, x_{i_{d}}^{(d)}\right)$, where $\mathbf{i} \in I=I_{1} \times \ldots \times I_{d}$. Hence, the grid values $u\left(x_{\mathbf{i}}\right)=u_{\mathbf{i}}$ belong to $\mathbb{R}^{I}$ which is the tensor space

$$
\mathbb{R}^{I}=\mathbb{R}^{I_{1}} \otimes \mathbb{R}^{I_{2}} \otimes \ldots \otimes \mathbb{R}^{I_{d}},
$$

i.e., $W_{j}=\mathbb{R}^{I_{j}}$ from (3.1) have the dimension $n_{j}:=\# I_{j}$. For simplicity we assume $n_{j}=n$ for all $1 \leq j \leq d$.

The representation of $w \in\rfloor_{R}$ needs a storage of $R d n$, while $w \in \mathcal{M}_{\mathbf{r}}$ with $\mathbf{r}=(r, \ldots, r)$ requires $r d n+r^{d}$ data.

\subsection{Application to matrices}

The index sets $I_{1}, \ldots, I_{d}$ and $J_{1}, \ldots, J_{d}$ give rise to the two tensor spaces $X:=\mathbb{R}^{I_{1}} \otimes \ldots \otimes \mathbb{R}^{I_{d}}$ and $Y:=\mathbb{R}^{J_{1}} \otimes \ldots \otimes \mathbb{R}^{J_{d}}$. Given matrices $A^{(j)} \in \mathbb{R}^{I_{j} \times J_{j}}(1 \leq j \leq d)$, its Kronecker product $\mathcal{A}:=A^{(1)} \otimes \ldots \otimes A^{(d)}$ is defined as the mapping

$$
\mathcal{A}: X \rightarrow Y, \quad x=x^{(1)} \otimes \ldots \otimes x^{(d)} \mapsto \mathcal{A} x=A^{(1)} x^{(1)} \otimes \ldots \otimes A^{(d)} x^{(d)} \in Y .
$$

\section{Discretisation and Numerical Solution of the Eigen- value Problem}

\subsection{Discretisation}

We apply the Galerkin approximation with respect to the tensor product piecewise linear basis functions. In the following, we make use of tensor representation of functions as well as the Kronecker tensor product representation of the elliptic operator $\Lambda$. Let us formulate the assumptions on the coefficients which ensure the respective tensor representation. 
We consider the elliptic operators of the form

$$
-\operatorname{div}(A \operatorname{grad} u)+\langle b, u\rangle+c u \equiv \Lambda_{A} u+\Lambda_{C} u,
$$

where $A=\left\{a_{i j}(x)\right\}_{i, j=1}^{d} \in \mathbf{L}_{\text {sym }}^{\infty}\left(\Omega, \mathbb{R}^{d \times d}\right), b \in L^{\infty}\left(\Omega, \mathbb{R}^{d}\right)$ and $c \in L^{\infty}(\Omega)$ satisfy the conditions

$$
\begin{aligned}
& 0<a_{\min }:=\inf _{x \in \Omega} \inf _{v \in \mathbb{C}^{d} \backslash\{0\}} \frac{\langle A(x) v, \bar{v}\rangle}{\langle v, \bar{v}\rangle} \leq \sup _{x \in \Omega} \sup _{v \in \mathbb{R}^{d} \backslash\{0\}} \frac{\langle A(x) v, \bar{v}\rangle}{\langle v, \bar{v}\rangle}=: a_{\max }<\infty \\
& 0 \leq-\frac{1}{2} \operatorname{div} b+c .
\end{aligned}
$$

To simplify the discussion, we further set $b=0$, though all the tensor constructions apply to this case as well. In addition, we make the following assumptions which lead to the low Kronecker rank representation of the discrete operator (the Galerkin stiffness matrix). We assume from the very beginning that the operator coefficients are separable, $a_{i j}, c \in \mathcal{C}_{R}$, with some moderate rank parameter $R \in \mathbb{N}$, i.e.,

$$
a_{i j}(x)=\sum_{k=1}^{R} a_{i j, k}^{(1)}\left(x_{1}\right) \cdots a_{i j, k}^{(d)}\left(x_{d}\right), \quad i, j=1, \ldots, d,
$$

and

$$
c(x)=\sum_{k=1}^{R} c_{k}^{(1)}\left(x_{1}\right) \cdots c_{k}^{(d)}\left(x_{d}\right) .
$$

Hence, in the case of rank-1 test and trial functions $u(x)=\prod_{\ell=1}^{d} u^{(\ell)}\left(x_{\ell}\right), v(x)=$ $\prod_{\ell=1}^{d} v^{(\ell)}\left(x_{\ell}\right)$, the associated bilinear forms can be written using the product ansatz as follows

$$
\begin{gathered}
\left\langle\Lambda_{A} u, v\right\rangle_{L^{2}}=\sum_{k=1}^{R} \sum_{i, j=1}^{d} \prod_{\ell=1}^{d}\left\langle a_{i j, k}^{(\ell)}\left(x_{\ell}\right) \frac{\partial^{\delta_{j \ell}}}{\partial x_{\ell}} u^{(\ell)}\left(x_{\ell}\right), \frac{\partial^{\delta_{i \ell}}}{\partial x_{\ell}} \bar{v}^{(\ell)}\left(x_{\ell}\right)\right\rangle_{L^{2}(\Omega)}, \\
\left\langle\Lambda_{C} u, v\right\rangle_{L^{2}}=\sum_{k=1}^{R} \prod_{\ell=1}^{d}\left\langle c_{k}^{(\ell)}\left(x_{\ell}\right) u^{(\ell)}\left(x_{\ell}\right), \bar{v}^{(\ell)}\left(x_{\ell}\right)\right\rangle_{L^{2}(\Omega)},
\end{gathered}
$$

where $\delta_{j i}$ is the Kronecker delta.

In the general case, a basis of piecewise polynomial functions

$$
\phi_{\mathbf{i}}(x)=\prod_{\ell=1}^{d} \phi_{i_{\ell}}\left(x_{\ell}\right), \quad \mathbf{i} \in \mathcal{I}=I^{d}:=\{1, \ldots, n\}^{d},
$$

can be used, where $\phi_{i_{\ell}}$ are low order polynomials in the variable $x_{\ell}$. For simplicity, we choose the Galerkin subspace $\mathbb{V} \subset\left(H_{0}^{1}(\Omega)\right)^{d}$ of piecewise linear basis functions (cf. Section 3.3). The Galerkin approximation to the eigenvalue problem takes the form

$$
\mathcal{L} U \equiv(\mathcal{A}+\mathcal{C}) U=\lambda \mathcal{M} U, \quad U \in \mathbb{R}^{\mathcal{I}}
$$


with the Kronecker tensor product representation (cf. Section 3.5)

$$
\mathcal{A}=\sum_{k=1}^{R} \sum_{i, j=1}^{d} \otimes_{\ell=1}^{d} A_{i j, k}^{(\ell)}, \quad \mathcal{C}=\sum_{k=1}^{R} \otimes_{\ell=1}^{d} C_{k}^{(\ell)}, \quad \mathcal{M}=\otimes_{\ell=1}^{d} M^{(\ell)}
$$

where $A_{i j, k}^{(\ell)}, C_{k}^{(\ell)}, M^{(\ell)} \in \mathbb{R}^{n \times n}$ are the tridiagonal matrices

$$
\begin{aligned}
A_{i j, k}^{(\ell)} & =\left\{\left\langle a_{i j, k}^{(\ell)}\left(x_{\ell}\right) \frac{\partial^{\delta_{j \ell}}}{\partial x_{\ell}} \phi_{p}, \frac{\partial^{\delta_{i \ell}}}{\partial x_{\ell}} \phi_{q}\right\rangle_{L^{2}}\right\}_{p, q=1}^{n}, \\
C_{k}^{(\ell)} & =\left\{\left\langle c_{k}^{(\ell)}\left(x_{\ell}\right) \phi_{p}, \phi_{q}\right\rangle_{L^{2}}\right\}_{p, q=1}^{n}, \quad M^{(\ell)}=\left\{\left\langle\phi_{p}, \phi_{q}\right\rangle_{L^{2}}\right\}_{p, q=1}^{n} .
\end{aligned}
$$

The following lemma concerns the complexity of this discretisation.

Lemma 4.1 The matrices $\mathcal{A}, \mathcal{C}$, and $\mathcal{M}$ have the respective Kronecker ranks $R d^{2}, R$, and 1 .

The storage requirements to represent these matrices scale linearly in the univariate problem size $n$,

$$
Q(\mathcal{A})=O\left(3 d^{3} R n\right), \quad Q(\mathcal{C})=O(3 d R n), \quad Q(\mathcal{M})=O(3 d n) .
$$

The same cost holds for the matrix-vector multiplication by a rank-1 vector.

Proof. The first assertion follows from the tridiagonal structure of the Kronecker factors in (4.7). Suppose that vector $U$ has the rank-1 tensor representation

$$
U=u^{(1)} \otimes \ldots \otimes u^{(d)}, \quad u^{(\ell)} \in \mathbb{R}^{n} .
$$

Then the matrix-times-vector multiplication with our stiffness matrices is reduced to onedimensional operations,

$$
\mathcal{A} U=\sum_{k=1}^{R} \sum_{i, j=1}^{d} \otimes_{\ell=1}^{d} A_{i j, k}^{(\ell)} u^{(\ell)}, \quad \mathcal{C} U=\sum_{k=1}^{R} \otimes_{\ell=1}^{d} C_{k}^{(\ell)} u^{(\ell)}, \quad \mathcal{M} U=\otimes_{\ell=1}^{d} M^{(\ell)} u^{(\ell)}
$$

which again leads to the linear cost in $n$.

Remark 4.2 Our approach is by no means limited to Galerkin discretisations. For example, in the case of the Laplace operator in $\mathbb{R}^{d}$ discretised by a finite difference scheme an a uniform product mesh, we obtain the simple Kronecker rank-d representation

$$
\mathcal{A}=A \otimes I_{n} \otimes \ldots \otimes I_{n}+I_{n} \otimes A \otimes I_{n} \ldots \otimes I_{n}+\ldots+I_{n} \otimes I_{n} \ldots \otimes A,
$$

where $A=\operatorname{tridiag}\{1,-2,1\} \in \mathbb{R}^{n \times n}$ and $I_{n}$ is the $n \times n$ identity matrix (cf. Section 3.5). 


\subsection{Iterative eigenvalue problem solvers with rank truncation}

Now we are able to discuss iterative methods accomplished with low rank truncation for solving the discrete eigenvalue problem as in (4.6).

Note that in the simplest case of finite difference discretisations (cf. Remark 4.2) the matrix $\mathcal{M}$ (in the general form as in (4.6)) is the identity matrix. For Galerkin discretisations, $\mathcal{M}$ is the mass matrix - however, the generalization of the power method to this generalized eigenvalue problem is standard. Note in addition that, since the mass matrix $\mathcal{M}$ has Kronecker rank one and, hence, it is diagonalisable at the expense $O(n \log n)$, so that (4.6) could be transformed to a standard eigenvalue problem.

Now consider the algebraic eigenvalue problem

$$
\mathcal{L} U=\lambda U, \quad U \in \mathbb{R}^{\mathcal{I}} .
$$

We are interested in the approximate solutions of (4.9) in the tensor class $\mathcal{M}_{\mathbf{r}} \subset \mathbb{R}^{\mathcal{I}}$. Notice, however, that the application of the operator $\mathcal{L}$ to an iterate $U^{(m)}$ which is of low-rank tensor format, in general, yields a result which has larger rank and is no longer contained in the class $\mathcal{M}_{\mathbf{r}}$. Hence, the application of $T_{\mathbf{r}}: \mathbb{R}^{\mathcal{I}} \rightarrow \mathcal{M}_{\mathbf{r}}$, i.e., the nonlinear truncation operator to $\mathcal{M}_{\mathrm{r}}$ defined in (3.5), to the result is essential for staying in the class $\mathcal{M}_{\mathbf{r}}$. On the other hand, this is the only modification of the standard power method and, conceptually, can be applied to the general class of iterative eigensolvers in the same fashion.

Largest eigenvalues. A standard method for computing the eigenpair $(\lambda, U)$ for the (single) largest eigenvalue is the simple power method. This includes the repeated matrixvector multiplication which in our approach is accomplished with the rank truncation,

$$
U^{(0)} \in \mathcal{M}_{\mathbf{r}}: \quad \widetilde{U}^{(m+1)}:=\mathcal{L} U^{(m)}, \quad U^{(m+1)}=T_{\mathbf{r}}\left(\widetilde{U}^{(m+1)}\right),
$$

so that $\left\|U^{(m+1)}\right\| /\left\|U^{(m)}\right\|$ will converge to the largest eigenvalue and $U^{(m)} /\left\|U^{(m)}\right\|$ to the associated eigenvector.

Lemma 4.3 Each step of the "truncated" power iteration needs a storage of size $Q_{P}=$ $O\left(3 R d^{3} r n+r^{d}\right)$. The same asymptotic complexity bound holds for the matrix-vector multiplication with rank-1 vectors.

Smallest eigenvalues. To compute the minimal eigenvalue, we apply the "truncated" power method (4.10) to the equivalent equation

$$
\mathcal{L}^{-1} U=\frac{1}{\lambda} U, \quad U \in \mathbb{R}^{n^{\otimes d}}
$$

In the present paper we study the effect of numerical methods designed in the rank structured tensor formats described above. In the context of equation (4.11), the power iteration applies to a class of elliptic operators that allow the explicit low Kronecker rank representation of the inverse matrix $\mathcal{L}^{-1}$.

In the more general situations, one can apply preconditioned truncated iterations to solve the equation

$$
\mathcal{L} V-U=0 \quad \text { to compute } \quad V=\mathcal{L}^{-1} U
$$


instead of evaluating the action of inverse matrix directly, $\mathcal{L}^{-1} U$, as follows. Given $U, V^{(0)} \in$ $\mathcal{M}_{\mathrm{r}}$ :

$$
\widetilde{V}^{(m+1)}:=V^{(m)}-\omega \mathcal{B}\left(\mathcal{L} V^{(m)}-U\right), \quad V^{(m+1)}=T_{\mathbf{r}}\left(\widetilde{V}^{(m+1)}\right) \rightarrow \mathcal{L}^{-1} U,
$$

where $\mathcal{B}$ can be chosen as the inverse of the shifted Laplacian (cf. [17] for the more detailed discussion of preconditionig issues). Likewise, one can apply the preconditioned inverse iteration to solve equation (4.11).

In the iteration (4.12) the truncation operator $T_{\mathbf{r}}$ can be applied to the residual vector $\mathcal{L} V^{(m)}-U$ and to the correction vector $\mathcal{B}\left(\mathcal{L} V^{(m)}-U\right)$, as well.

\section{$5 \quad$ Error analysis for elliptic eigenvalue problems}

In this section, we will derive error estimates for our low tensor-rank approximation for elliptic eigenvalue problems of the form $(1.1 \mathrm{~b})$. We further emphasize that numerical experiments (see [6]) show that the applicability of our method is by no means limited to this problem class but also performs efficiently for problems in quantum chemistry as, e.g., for solutions of the Hartree-Fock equation [19].

We will prove that, for the general eigenvalue problem of the form (1.1) in $\Omega=\prod_{i=1}^{d}\left(a_{i}, b_{i}\right), d=2,3$, the low tensor-rank approximation converges at the same rate as in the one-dimensional case, $d=1$, while the cost increases only linearly (up to logarithmic terms) with respect to the number of univariate unknowns, $n$, and the spatial dimension. The proof is structured as follows. In Subsection 5.1, we will estimate the error of (global) polynomial tensor interpolation of entire functions $u$ in terms of the growth of its derivatives. In Subsection 5.2, the derivatives of the eigensolutions in (1.1) will be estimated which in turn complete the polynomial interpolation estimates. Finally, in Subsection 5.3 we will project this approximation to the usual $\mathbb{P}_{1}$-finite element on a tensor mesh of step size $h$ and show that the usual linear convergence rates are preserved.

\subsection{Polynomial approximation of analytic functions}

To understand the separability property of eigenfunctions we analyse their regularity with respect to some classes of functions which allow the holomorphic extension to the complex plane.

The error estimates will be derived for the function set $\mathcal{A}_{M, \rho}(I)$ and for its multidimensional counterpart $\mathcal{A}_{M, \rho}\left(I^{d}\right), d \geq 2$. The definition of the space $\mathcal{A}_{M, \rho}\left(I^{d}\right)$ requires several steps. For the interval $I:=(-1,1)$ and $\rho>1$, Bernstein's regularity ellipse is given by (cf. $[2])$

$$
\mathcal{E}_{\rho}:=\left\{z \in \mathbb{C}:|z-1|+|z+1| \leq \rho+\rho^{-1}\right\} .
$$

Its semi-axes are $a=\frac{\rho+\rho^{-1}}{2}$ and $b=\frac{\rho-\rho^{-1}}{2}$, implying $a+b=\rho$.

Definition 5.1 Let $I=(-1,1)$ and $M>0, \rho>1$ be given constants. $\mathcal{A}_{M, \rho}(I)$ is the class of functions $f \in C^{\infty}(I)$ having a holomorphic extension to $\mathcal{E}_{\rho}(I)$ such that

$$
|f(z)| \leq M \quad \forall z \in \mathcal{E}_{\rho}(I) .
$$


Next, we introduce the multidimensional analogue of $\mathcal{A}_{M, \rho}(I)$ on the tensor domain $I^{d}:=(-1,1)^{d}$. Let $\mathcal{E}_{\rho}^{(j)}:=I \times \ldots \times I \times \mathcal{E}_{\rho} \times I \times \ldots \times I$ with $\mathcal{E}_{\rho}$ to be inserted at the $j$ th position.

Definition 5.2 For given constants $M>0, \rho>1$, the set $\mathcal{A}_{M, \rho}\left(I^{d}\right)$ consists of all functions $f \in C^{\infty}\left(I^{d}\right)$ having holomorphic extensions to $\mathcal{E}_{\rho}^{(j)}$, for all $1 \leq j \leq d$, and satisfying

$$
\max _{1 \leq j \leq d}\left\{\sup _{x \in \mathcal{E}_{\rho}^{(j)}}|f(x)|\right\} \leq M .
$$

The following remark recalls the well-known fact that controlling all higher derivatives of a function implies that it belongs to $\mathcal{A}_{M, \rho}(I)$ (see e.g. [22] for the proof). For a Lipschitz domain $\Omega \subset \mathbb{R}^{d}$ and $\ell \in \mathbb{N}$, we define the norms

$$
\left\|\nabla^{\ell} u\right\|_{L^{2}(\Omega)}^{2}:=\sum_{|\alpha|=\ell} \frac{\ell !}{\alpha !}\left\|D^{\alpha} u\right\|_{L^{2}(\Omega)}^{2} \quad \text { and } \quad\left\|\nabla^{\ell} u\right\|_{L^{\infty}(\Omega)}:=\left\|\sqrt{\sum_{|\alpha|=\ell} \frac{\ell !}{\alpha !}\left|D^{\alpha} u\right|^{2}}\right\|_{L^{\infty}(\Omega)} .
$$

For $u$ replaced by vectors or matrices, the absolute value $|\cdot|$ is to be replaced by the Euclidean or spectral norm.

Remark 5.3 Assume that a function $u: I \rightarrow \mathbb{R}$ satisfies for some $C_{u}, \gamma_{u} \geq 0$

$$
\left\|\frac{\partial^{p} u}{\partial x^{p}}\right\|_{L^{\infty}(I)} \leq C_{u} \gamma_{u}^{p} p ! \quad \text { for all } p \in \mathbb{N}_{0} .
$$

Then $u \in \mathcal{A}_{M, \rho}(I)$ holds with $\rho=1+\gamma_{u}^{-1}>1, M=C \cdot C_{u}$. Definition 5.2 directly implies the following conclusion:

$$
\left\|\nabla^{p} u\right\|_{L^{\infty}\left(I^{d}\right)} \leq C_{u} \gamma_{u}^{p} p ! \quad \text { for all } p \in \mathbb{N}_{0}, \quad \text { and } \quad u \in \mathcal{A}_{M, \rho}\left(I^{d}\right)
$$

with constants $\rho, M$ as in (5.1).

For the continuous multivariate functions $f=f\left(x_{1}, \ldots, x_{d}\right): \mathbb{R}^{d} \rightarrow \mathbb{R}$, we use the tensor product interpolant

$$
\mathbf{I}_{N} f=I_{N}^{1} \ldots I_{N}^{d} f \in P_{N}\left[I^{d}\right]
$$

where $I_{N}^{i} f(1 \leq i \leq d)$ denotes the interpolation polynomial of degree $N$ with respect to the variables $x_{i} \in I:=[-1,1]$ interpolating $f$ with respect to the variables $x_{i} \in I:=[-1,1]$ at the Chebyshev nodes.

Proposition 5.4 Let $M>0$ and $\rho>1$ be given. For all $f \in \mathcal{A}_{M, \rho}\left(I^{d}\right)$ and $N>1$ the estimate

$$
\left\|f-\mathbf{I}_{N} f\right\|_{L^{2}\left(I^{d}\right)} \leq c M(\log N)^{d} \rho^{-N}
$$

holds.

The corresponding result for the $H^{m}-$ norm, $m \in \mathbb{N}$, reads as: For each $1<\rho_{1}<\rho$,

$$
\left\|f-\mathbf{I}_{N} f\right\|_{H^{m}\left(I^{d}\right)} \leq C M N^{m}(\log N)^{d} \rho_{1}^{-N} .
$$

Hence, by an appropriate diminishment of $\rho$, e.g., $\rho \leftarrow \frac{\rho+1}{2}$, we may select $\rho_{1}=\rho$.

Proof. The bound (5.3) was justified in [12]. Its conterpart related to $H^{m}$-norms, (5.4) is based on the respective results in [31].

In the next section we derive the regularity results for solutions of elliptic eigenvalue problems which will imply Remark 5.3. 


\subsection{Regularity for elliptic eigenvalue problems with smooth data}

Consider the eigenvalue problem for the differential operator

$$
\Lambda u:=-\nabla \cdot(A \nabla u)+\langle b, \nabla u\rangle+c u,
$$

where $\langle a, b\rangle:=\sum_{i=1}^{d} a_{i} b_{i}$ (without complex conjugation). We say that $\Lambda$ is uniformly elliptic if $A \in \mathbf{L}_{\mathrm{sym}}^{\infty}\left(\Omega, \mathbb{R}^{d \times d}\right), b \in \mathbf{L}^{\infty}\left(\Omega, \mathbb{R}^{d}\right)$, and $c \in L^{\infty}(\Omega)$ satisfy the conditions in (4.2). We assume that the coefficients of the operator $\Lambda$ are analytic; i.e., there exist positive constants $C_{\mathcal{A}}, C_{b}, C_{c}$ and $\gamma_{\mathcal{A}}, \gamma_{b}, \gamma_{c}$ such that

$$
\begin{aligned}
\left\|\nabla^{p} A\right\|_{L^{\infty}(\Omega)} & \leq C_{\mathcal{A}} \gamma_{\mathcal{A}}^{p} p ! \quad \forall p \in \mathbb{N}_{0}, \\
\left\|\nabla^{p} b\right\|_{L^{\infty}(\Omega)} & \leq C_{b} \gamma_{b}^{p} p ! \\
\left\|\nabla^{p} c\right\|_{L^{\infty}(\Omega)} & \leq C_{c} \gamma_{c}^{p} p ! \quad \forall p \in \mathbb{N}_{0},
\end{aligned}
$$

We consider two types of domain: Either $\Omega=\mathbb{R}^{d}$ or $\Omega$ is a bounded Lipschitz domain with analytic boundary, i.e., there is a finite family $\mathcal{U}$ of open subset in $\mathbb{R}^{d}$ along a family of bijective maps ${ }^{1}\left\{\chi_{U}: \overline{B_{1}} \rightarrow \bar{U}\right\}_{U \in \mathcal{U}}$ such that

$$
\begin{array}{ll}
\forall U \in \mathcal{U}: & \chi_{U} \in C^{0,1}\left(\overline{B_{1}}, \bar{U}\right), \quad \chi_{U}^{-1} \in C^{0,1}\left(\bar{U}, \overline{B_{1}}\right), \\
\forall U \in \mathcal{U}: & \chi_{U}\left(B_{1}^{0}\right)=U \cap \partial \Omega, \quad \chi_{U}\left(B_{1}^{+}\right)=U \cap \Omega, \quad \chi_{U}\left(B_{1}^{-}\right)=U \cap \mathbb{R}^{d} \backslash \bar{\Omega}, \\
\exists C_{\Gamma}, \gamma_{\Gamma} \quad \forall U \in \mathcal{U}: & \left\|\nabla^{p} \chi_{U}\right\|_{L^{\infty}\left(B_{1}\right)} \leq C_{\Gamma} \gamma_{\Gamma}^{p} p ! \quad \forall p \in \mathbb{N}_{0} .
\end{array}
$$

Let us consider the eigenvalue problem: Find a pair $(\lambda, u) \in \mathbb{R} \times H_{0}^{1}(\Omega) \backslash\{0\}$ such that

$$
\begin{aligned}
& \Lambda u=\lambda u \text { in } \Omega, \\
& u=0 \quad \text { on } \partial \Omega
\end{aligned}
$$

with $\Lambda$ as in (5.5). Let $E(\lambda)$ denote the eigenspace for the eigenvalue $\lambda$.

Theorem 5.5 Let $\Omega$ be an analytic, bounded Lipschitz domain which satisfies (5.7). Assume that the coefficients $A, b, c$ satisfy (5.6). Then, any eigenfunction $u \in E(\lambda)$ of (5.8) (normalised to $\|u\|_{L^{2}(\Omega)}=1$ ) is analytic. There exist constants $C, K>0$ depending only on the constants in (5.6), (5.7), and on $a_{\min }$ and the spatial dimension d such that

$$
\left\|\nabla^{p+2} u\right\|_{L^{2}(\Omega)} \leq C K^{p+2} \max \{p, \sqrt{|\lambda|}\}^{p+2} \quad \text { for all } p \geq 0 .
$$

Proof. The statement follows from [22, Theorem 5.3.10] as follows. First, let $|\lambda| \geq 1$ and consider (5.8) as the equation

$$
-\varepsilon^{2} \nabla \cdot(A \nabla u)+\langle\tilde{b}, \nabla u\rangle+(\tilde{c}-1) u=f \quad \text { in } \Omega \quad \text { with }\left.\quad u\right|_{\partial \Omega}=0
$$

where $\varepsilon^{2}=\lambda^{-1}, \tilde{b}=\lambda^{-1} b, \tilde{c}=c / \lambda$ and $f \equiv 0$. For the quantity $\mathcal{E}$ in [22, Theorem 5.3.10] we obtain the estimate

$$
\mathcal{E}^{-1}:=C_{b}+\frac{\sqrt{1+C_{c} /|\lambda|}}{|\lambda|^{-1 / 2}}+1 \leq 1+C_{b}+\sqrt{|\lambda|+C_{c}} \leq C_{1} \sqrt{|\lambda|},
$$

\footnotetext{
${ }^{1} B_{1}$ denotes the unit ball in $\mathbb{R}^{d}$ and $B_{1}^{0}:=\left\{x \in B_{1} \mid x_{d}=0\right\}$. For $\sigma \in\{+,-\}$, we set $B_{1}^{\sigma}:=$ $\left\{x \in B_{1} \mid \sigma x_{d}>0\right\}$.
} 
where $C_{1}:=1+C_{b}+\sqrt{1+C_{c}}$. The other quantities which appear in [22, Theorem 5.3.10] have to be substituted by

$$
C_{f} \leftarrow 0, \quad C_{c} \leftarrow C_{c}+1, \quad \mathcal{E} \leftarrow C_{2}|\lambda|^{-1 / 2}, \quad\left(\frac{\mathcal{E}}{\varepsilon}\right)^{2} \leftarrow C_{2}^{2}
$$

with $C_{2}:=\left(\sqrt{1+C_{c}}+C_{b}\right)^{-1}$. From (4.2) we conclude that

$$
\begin{aligned}
\operatorname{Re} a(u, u) & =\int_{\Omega}\langle A \nabla u, \nabla \bar{u}\rangle+\operatorname{Re}(\langle b, \nabla u\rangle \bar{u})+c|u|^{2} \\
& =\int_{\Omega}\langle A \nabla u, \nabla \bar{u}\rangle+\frac{1}{2}\left\langle b, \nabla\left(|u|^{2}\right)\right\rangle+c|u|^{2} \\
& =\int_{\Omega}\langle A \nabla u, \nabla \bar{u}\rangle+\left(-\frac{1}{2} \operatorname{div} b+c\right)|u|^{2} \\
& \stackrel{(4.2)}{\geq} a_{\min }\|\nabla u\|_{L^{2}(\Omega)}^{2}
\end{aligned}
$$

holds. Since $u$ is an eigenfunction corresponding to $\lambda$ and $\|u\|_{L^{2}(\Omega)}=1$ we obtain

$$
\|\nabla u\|_{L^{2}(\Omega)} \leq a_{\min }^{-1 / 2} \sqrt{\operatorname{Re} a(u, u)}=\sqrt{(\operatorname{Re} \lambda) / a_{\min }}
$$

Plugging these quantities into the estimate in [22, Theorem 5.3.10] we get

$$
\left\|\nabla^{p+2} u\right\|_{L^{2}(\Omega)} \leq C K^{p+2} \max \{p, \sqrt{|\lambda|}\}^{p+2}
$$

where $C$ only depends on the constants $C_{\mathcal{A}}, C_{b}, C_{c}, \gamma_{\mathcal{A}}, \gamma_{b}, \gamma_{c}, C_{\Gamma}, \gamma_{\Gamma}, a_{\text {min }}$. As explained in [22, Remark 5.3.11] the coercivity assumption which is imposed in [22, Theorem 5.3.10] is not required for this estimate. The proof of [22, Theorem 5.3.10] covers only the case $d=2$. However, the only part therein, where $d=2$ (instead of general $d$ ) is used explicitly, is the mapping lemma [22, Lemma 4.3.1]. Inspection of the proof shows that the case $d \geq 3$ can be handled analogously while, then, the constants in (5.9) in general depend also on the spatial dimension $d$.

The case $|\lambda|<1$ is even simpler because we consider

$$
-\varepsilon^{2} \nabla \cdot(A \nabla u)+\langle b, \nabla u\rangle+(c-\lambda) u=f \quad \text { in } \Omega \quad \text { with }\left.\quad u\right|_{\partial \Omega}=0
$$

where $f=0$ and $\varepsilon^{2}=1$. By repeating the steps in the first part of the proof with coefficients $\tilde{b}=b, \tilde{c}=c-\lambda$ with $|\lambda|<1$ we obtain

$$
\left\|\nabla^{p+2} u\right\|_{L^{2}(\Omega)} \leq C(p K)^{p+2} .
$$

Remark 5.6 Let $\Omega=\mathbb{R}^{d}$. Assume that the coefficients A,b,c satisfy (5.6). Then the estimate

$$
\left\|\nabla^{p+2} u\right\|_{L^{2}\left(\mathbb{R}^{d}\right)} \leq C K^{p+2} \max \{p, \sqrt{|\lambda|}\}^{p+2} \quad \text { for all } p \geq 0
$$

follows from [22, Prop. 5.5.1] by a simple repetition of the arguments of the previous proof. 
Now we are in a position to apply the regularity results in Theorem 5.5 to derive the low separation rank approximations to certain class of eigenfunctions in (5.8).

Corollary 5.7 Let $\Omega=(-\beta, \beta)^{d}$ be some fixed hypercube in $\mathbb{R}^{d}$ or let $\Omega=\mathbb{R}^{d}$. Assume that the coefficients A,b,c satisfy (5.6). Then the estimate (5.2) holds in $\Omega$ with

$$
\rho=1+\frac{c}{1+\sqrt{|\lambda|}}>1 \quad M=\frac{C \tilde{C}_{d}}{\sqrt{2 \pi}}(K(p+\sqrt{|\lambda|}))^{k}
$$

for $k=\lceil(d+1) / 2\rceil$ and with $\tilde{c}_{d}, \tilde{C}>0$ depending only on the constants $C, K$ in Theorem 5.5 and the dimension $d$.

Proof. To prove the assertion we check that the bound (5.9) implies Remark 5.3 with the respective constants $C_{u}, \gamma_{u}$, where $u, \lambda$ is the eigenpair in (5.8). Due to the Sobolev embedding theorem, the $L^{2}$-bound (5.9) implies the corresponding estimate in $L^{\infty}$ norm for $k=\left\lceil\frac{d+1}{2}\right\rceil$

$$
\begin{aligned}
\left\|\nabla^{p+2} u\right\|_{L^{\infty}(B)} & \leq C_{d}\left\|\nabla^{p+2} u\right\|_{H^{k}(\Omega)} \stackrel{(5.9)}{\leq} C_{d} K^{p+k+2} \max \{p+k, \sqrt{|\lambda|}\}^{p+k+2} \\
& \leq \tilde{C}_{d}(K(p+\sqrt{|\lambda|}))^{k} K^{p+2} \max \{p, \sqrt{|\lambda|}\}^{p+2} .
\end{aligned}
$$

Since the derivation of the sharp dependence of the error on the size of the eigenvalues is not our goal, we simply estimate by Stirling's formula

$$
\left\|\nabla^{p+2} u\right\|_{L^{\infty}(B)} \leq C_{u} \gamma_{u}^{p+2}(p+2) !
$$

with $C_{u}=\tilde{C}_{d}(K(p+\sqrt{|\lambda|}))^{k} / \sqrt{2 \pi}$ and $\gamma_{u}=\mathrm{e}(1+\sqrt{|\lambda|}) K$. Hence, from Proposition 5.4 the estimate (5.10) follows.

Now we are able to derive the separable approximations for a class of elliptic eigenvalue problems.

Theorem 5.8 Let the assumptions of Corollary 5.7 be satisfied. Then, there exists an element $u_{\mathbf{r}} \in \mathcal{M}_{\mathbf{r}}$ with $\mathbf{r}=(r, \ldots, r)$, such that for the eigenfunction in (5.8) we have

$$
\left\|u-u_{\mathbf{r}}\right\|_{H^{1}(\Omega)} \leq c M r(\log r)^{d} \rho^{-r}
$$

where $\rho$ and $M$ are as in Corollary 5.7. The related representation of $u_{\mathbf{r}}$ in $\mathcal{C}_{R}$ has at most $\operatorname{rank} R=r^{d-1}$.

Proof. Corollary 5.7 ensures that for the eigenfunction we have $u \in \mathcal{A}_{M, \rho}(\Omega)$ with the respective constants $M, \rho$. Hence, we apply the tensor product interpolant $\mathbf{I}_{r-1} u=I_{r-1}^{1} \ldots I_{r-1}^{d} u \in$ $\mathbb{P}_{r-1}$ on $\Omega$ with respect to $d$ variables as in Proposition 5.4 and obtain the bound (5.3). The representation in the format $\mathcal{C}_{R}$ is obtained by reordering of rank- 1 summands in the Tucker decomposition. This completes our proof.

Remark 5.9 By choosing

$$
r \geq C \sqrt{|\lambda|} \log \frac{1}{\varepsilon}
$$

and taking into account (5.10), the bound in (5.11) implies

$$
\left\|u-u_{\mathbf{r}}\right\|_{H^{1}(\Omega)} \leq C \varepsilon .
$$




\subsection{Approximations in tensorised $\mathbb{P}_{1}$-finite element spaces}

Theorem 5.8 shows that in the tensor manifold $\mathcal{M}_{\mathbf{r}}, \mathbf{r}=(r, r, \ldots, r)$, there exists a function $u_{\mathbf{r}}$ which approximates the eigenfunction of the elliptic problem (1.1) with converges exponentially with respect to $r$.

The representative $u_{\mathbf{r}}$ is a global polynomial. In this section we will show that, also in the tensorised low order $\mathbb{P}_{1}$-finite element space on a tensor mesh (satisfying zero-boundary conditions) with step size $h$, there exists a representative with converges linearly in $h$. We set $\mathbb{V}_{\mathbf{r}}:=\bigotimes_{i=1}^{d} \mathbb{P}_{r-1}$ and choose some basis $\left(\phi_{k}\right)_{k=0}^{r-1}$ for $\mathbb{P}_{r-1}$. Let $\mathcal{I}_{h}$ denote the interpolation of $H_{0}^{1}(I)$ on the $\mathbb{P}_{1}$-finite element space on a mesh of $I$ with maximal step size $h$. Let

$$
\phi_{k, h}:=\mathcal{I}_{h} \phi_{k} \quad 0 \leq k \leq r-1
$$

and let $V_{r, h}:=\operatorname{span}\left\{\phi_{k, h}: 0 \leq k \leq r-1\right\}$. Then, the space $\mathbb{V}_{\mathbf{r}, h}=\bigotimes_{i=1}^{d} V_{r, h}$ is a subset of $\mathcal{M}_{\mathbf{r}}$ and we will prove that there is an element $u_{\mathbf{r}, h}:=I_{h} u_{\mathbf{r}} \in \mathbb{V}_{\mathbf{r}, h}$ which satisfies

$$
\left\|u_{\mathbf{r}}-I_{h} u_{\mathbf{r}}\right\|_{H^{1}(\Omega)} \leq C h
$$

Theorem 5.10 Let $\Omega=(-\beta, \beta)^{d}$ be some fixed hypercube in $\mathbb{R}^{d}$. Assume that the coefficients $A, b, c$ satisfy (5.6). Then, there exists an element $u_{\mathbf{r}, h} \in \mathbb{V}_{\mathbf{r}, h} \subset \mathcal{M}_{\mathbf{r}}$ with $\mathbf{r}=(r, \ldots, r)$, such that for the eigenfunction in (5.8) we have

$$
\left\|u-u_{\mathbf{r}, h}\right\|_{H^{1}(\Omega)} \leq c h
$$

where $\rho$ and $M$ are as in Corollary 5.7. The related representation of $u_{\mathbf{r}, h}$ in $\mathcal{C}_{R}$ has at most $\operatorname{rank} R=r^{d-1}$.

Proof. Let $u, u_{\mathbf{r}}$ be as in (5.11) and let $u_{\mathbf{r}, h}:=I_{h} u_{\mathbf{r}} \in \mathbb{V}_{\mathbf{r}, h}$. Then, by the triangle inequality and Theorem 5.8 we have

$$
\left\|u-u_{\mathbf{r}, h}\right\|_{H^{1}(\Omega)} \leq c M r(\log r)^{d} \rho^{-r}+\left\|u_{\mathbf{r}}-I_{h} u_{\mathbf{r}}\right\|_{H^{1}(\Omega)} .
$$

Standard interpolation results lead to

$$
\left\|u_{\mathbf{r}}-I_{h} u_{\mathbf{r}}\right\|_{H^{1}(\Omega)} \leq C h\left\|u_{\mathbf{r}}\right\|_{H^{2}(\Omega)} .
$$

Now, from (5.4), Theorem 5.5 and Remark 5.6, we conclude that

$$
\left\|u_{\mathbf{r}}\right\|_{H^{2}(\Omega)} \leq\|u\|_{H^{2}(\Omega)}+\left\|u-u_{\mathbf{r}}\right\|_{H^{2}(\Omega)} \leq C K^{2}|\lambda|+c M r^{2}(\log r)^{d} \rho^{-r} .
$$

The second summand is bounded uniformly in $r$ so that the assertion follows by combining these estimates.

Finally, we quote a standard error estimate for the Galerkin discretisation of eigenvalue problems. 
Theorem 5.11 Assume $r$ in $\mathcal{M}_{\mathbf{r}}$ with $\mathbf{r}=(r, r, \ldots, r)$ is chosen sufficiently large and $h$ in the definition of $\mathbb{V}_{\mathbf{r}, h}$ be chosen sufficiently small. Let the geometric and algebraic multiplicity of a continuous eigenvalue $\lambda_{0}$ coincide. For any $e \in E(\lambda)$ with $\|e\|_{L^{2}(\Omega)}=1$, there exists a discrete eigenfunction in $e_{\mathbf{r}} \in \mathbb{V}_{\mathbf{r}} \subset \mathcal{M}_{\mathbf{r}}$ resp. $e_{\mathbf{r}, h} \in \mathbb{V}_{\mathbf{r}, h} \subset \mathcal{M}_{\mathbf{r}}$ such that

$$
\begin{aligned}
\left\|e-e_{\mathbf{r}}\right\|_{H^{1}(\Omega)} & \leq C M r(\log r)^{d} \rho^{-r} \\
\left\|e-e_{\mathbf{r}, h}\right\|_{H^{1}(\Omega)} & \leq C h .
\end{aligned}
$$

The proof of this theorem can be found in [11, Theorem 11.2.20]. The restriction to simple eigenvalues for the eigenvector error estimates is quite strong. The error estimates have been generalized for self-adjoint problems in [20] and [25] to the case of multiple and also clustered eigenvalues. For the non-selfadjoint case we refer to [1].

To conclude this section we observe that for a special class of spectral problems, Theorem 5.8 can be applied in the finite hypercube $\Omega$ defined above. Specifically, we assume that the eigenfunction of problem (5.8) with $\Omega=\mathbb{R}^{d}$ exhibits the exponential decay,

$$
|u(x)| \leq C e^{-\alpha\|x\|} \quad \text { as } \quad x \rightarrow \infty .
$$

Given $\varepsilon>0$, consider the "approximating" eigenvalue problem posed in the hypercube $\Omega$ of side length $\beta=O(|\log \varepsilon|)$, with the corresponding eigenfunction $u_{\beta}$, and suppose that $\left\|u-u_{\beta}\right\| \leq C \varepsilon$.

Remark 5.12 Corollary 5.7 ensures that the eigenfunction $u_{\beta}$ allows the same upper bound on the Tucker and canonical ranks as in Theorem 5.8. Hence, in this case the truncated iteration can be applied directly to the problem in finite domain $\Omega_{\beta}$.

\section{$6 \quad$ Numerics and concluding remarks}

In this section we present numerical illustrations for $2 \mathrm{D}$ and $3 \mathrm{D}$ eigenvalue problems. In particular, we present numerical examples for the "truncated" power iteration applied to the inverse of an elliptic operator $\mathcal{L}^{-1}$, where we set $b=0$. In the case of constant/separable coefficients , $\mathcal{L}^{-1}$ will be approximated by a low rank Kronecker product, e.g., obtained from the sinc-quadrature method $[10,12,16]$.

\subsection{Operators with constant coefficients}

First we consider the finite difference analogue of the negative Laplacian $\mathcal{A}$ on the domain $\Omega=(0,1)^{d}$, as in $(4.8)$ for $d=2,3$ with zero boundary conditions. The case $d=2$ is of interest since in that case the projection operator $T_{\mathbf{r}}$ onto tensor structured manifold can easily be realised by the "truncated" SVD algorithm.

We recall that the eigenvalues are

$$
\lambda_{\mathbf{i}}=(n+1)^{2} \sum_{\ell=1}^{d} 4 \sin ^{2} \frac{\pi i_{\ell}}{2(n+1)}, \quad \mathbf{i} \in \mathbb{N}^{d} \text { with components } 1 \leq i_{\ell} \leq n,
$$


and the corresponding eigenvectors are exact rank-1 tensors:

$$
U_{\mathbf{i}}=u^{\left(i_{1}\right)} \otimes \ldots \otimes u^{\left(i_{d}\right)},
$$

where $u^{\left(i_{\ell}\right)} \in \mathbb{R}^{n}$ with entries

$$
u_{s}^{\left(i_{\ell}\right)}=\sin \frac{\pi i_{\ell} s}{n+1}, \quad 1 \leq s \leq n .
$$

We implement the power iteration for the matrix $\mathcal{L}=\mathcal{A}^{-1}$, which is (approximately) represented in the rank- $R$ Kronecker format in the form

$$
\mathcal{L} \approx \mathcal{L}_{R}:=\sum_{k=-M}^{M} c_{k} \bigotimes_{\ell=1}^{d} \exp \left(-t_{k} A^{(\ell)}\right) \approx \mathcal{A}^{-1}, \quad R=2 M+1, \quad t_{k}, c_{k}>0
$$

with $A^{(\ell)}:=\operatorname{tridiag}\{-1,2,-1\} \in \mathbb{R}^{n \times n}$, providing exponential convergence in $R$ (see [12]). Specifically, we take

$$
t_{k}=e^{k \mathfrak{h}}, \quad c_{k}=\mathfrak{h} t_{k}, \quad \mathfrak{h}=\pi / \sqrt{M},
$$

which leads to the convergence rate

$$
\left\|\mathcal{A}^{-1}-\mathcal{L}_{R}\right\| \leq C e^{-\pi \sqrt{M}}, \quad R=2 M+1 .
$$

For even better coefficients $t_{k}, c_{k}$ compare [5]. We recall that the memory requirements for this algorithm are linear in $n$, i.e. $\mathcal{O}(d R n)$, while a linear complexity in the number of grid points in the volume would lead to $\mathcal{O}\left(n^{d}\right)$.

The matrix-vector multiplication of $\mathcal{L}_{R}$ with a rank-1 vector in $\mathbb{R}^{n^{\otimes d}}$ takes $\mathcal{O}(d R n \log n)$ operations by using the diagonalisation

$$
\exp \left(-t_{k} A^{(\ell)}\right)=F_{s}^{\prime} \cdot D \cdot F_{s}, \quad D=\operatorname{diag}\left\{e^{-t_{k} \lambda_{1}}, \ldots, e^{-t_{k} \lambda_{n}}\right\}
$$

where $F_{s}$ is the sin-transform matrix of size $n$, and $\lambda_{i}(i=1, \ldots, n)$ are the respective eigenvalues of the 1D Laplacian.

Example 1. We present the results for $\lambda=\lambda_{\min }$ in the 2D case, computed in MATLAB by using Intel $(R)$ T230/1.66 GHz processor. Since the eigenvector has rank one, we apply at each iteration step the truncation operator $T_{1}$ implemented via truncated SVD. In the next table we present numerical illustrations for a sequence of grids indicating the $\mathrm{CPU}$ time (sec.) for one matrix-vector multiplication with $\mathcal{L}_{R} \approx \mathcal{A}^{-1}$, (with fixed parameter $M=10$, the respective Kronecker rank of $\mathcal{L}_{R}$ is $R=2 M+1=21$ ), and accomplished with the rank truncation applied to each iterand $\mathcal{L}_{R} U^{(m)}$. We present the number of power iterations on each grid level, as well as the resulting relative errors $\delta_{\lambda}=\left|\lambda-\lambda_{h, R}\right| /|\lambda|$ and $\delta_{u}=\left\|U-U_{h, R}\right\|_{1} /\|U\|_{1}$.

The results indicate the true asymptotical convergence of the truncated iteration in the mesh parameter $h=\pi /(n+1)$, on a sequence of large $n \times n$ grids for $n=2^{p}-1, p=$ $8,10, \ldots, 16$. Table 6.1 also indicates perfect linear scaling of the tensor method in $n$ (compare with $\mathcal{O}\left(n^{2}\right)$ for traditional methods of linear complexity). The number of power iterations increases as $O(\log n)$ as expected. 


\begin{tabular}{c|c|c|c|c}
$n \times n$ grid size & Time $/$ it. & $\delta_{\lambda}$ & $\delta_{u}$ & it. \\
\hline $255 \times 255$ & 0.04 & $7.3 \cdot 10^{-6}$ & $5.0 \cdot 10^{-4}$ & 5 \\
\hline $1023 \times 1023$ & 0.1 & $2.5 \cdot 10^{-7}$ & $1.1 \cdot 10^{-4}$ & 6 \\
\hline $4047 \times 4047$ & 0.4 & $1.0 \cdot 10^{-8}$ & $2.0 \cdot 10^{-5}$ & 7 \\
\hline $16191 \times 16191$ & 1.45 & $3.8 \cdot 10^{-10}$ & $4.2 \cdot 10^{-6}$ & 8 \\
\hline $64767 \times 64767$ & 7.0 & $2.2 \cdot 10^{-11}$ & $1.17 \cdot 10^{-6}$ & 9
\end{tabular}

Table 6.1: Minimal eigenvalue for 2D Laplacian.

Example 2. We consider the tensor computation of several smallest eigenvalues using the same tensor power iteration, but accomplished with successive Gram-Schmidt orthogonalisation at each iteration step. We again solve the problem on a sequence of large $n \times n$ grids for $n=2^{p}-1, p=6,8, \ldots, 16$. The number of power iterations observed is about $2 \leq i t \leq 4$ for all grids with $p \geq 8$. Here the computation of the initial guess by means of a nested iteration involving coarser grids is very helpful. Table 6.1 presents the iteration history for three eigenpairs $\left(\lambda_{i}, u_{i}\right)(i=1,2,3)$.

\begin{tabular}{c|c|c|c|c|c|c|c}
$p$ & Time/it. & $\delta_{\lambda_{1}}$ & $\delta_{u_{1}}$ & $\delta_{\lambda_{2}}$ & $\delta_{u_{2}}$ & $\delta_{\lambda_{3}}$ & $\delta_{u_{3}}$ \\
\hline 6 & 0.02 & $2.3 \cdot 10^{-6}$ & $2.5 \cdot 10^{-4}$ & $2.7 \cdot 10^{-5}$ & $1.9 \cdot 10^{-3}$ & $4.2 \cdot 10^{-6}$ & $3.3 \cdot 10^{-3}$ \\
\hline 8 & 0.03 & $2.1 \cdot 10^{-7}$ & $8.9 \cdot 10^{-5}$ & $1.3 \cdot 10^{-6}$ & $3.9 \cdot 10^{-4}$ & $5.3 \cdot 10^{-7}$ & $1.1 \cdot 10^{-3}$ \\
\hline 10 & 0.1 & $1.7 \cdot 10^{-8}$ & $2.2 \cdot 10^{-5}$ & $6.9 \cdot 10^{-8}$ & $9.4 \cdot 10^{-5}$ & $1.3 \cdot 10^{-8}$ & $1.8 \cdot 10^{-4}$ \\
\hline 12 & 0.39 & $9.9 \cdot 10^{-10}$ & $5.2 \cdot 10^{-6}$ & $4.5 \cdot 10^{-9}$ & $2.3 \cdot 10^{-5}$ & $1.9 \cdot 10^{-9}$ & $7.0 \cdot 10^{-5}$ \\
\hline 14 & 1.6 & $6.5 \cdot 10^{-11}$ & $1.3 \cdot 10^{-6}$ & $2.8 \cdot 10^{-10}$ & $6.0 \cdot 10^{-6}$ & $1.5 \cdot 10^{-10}$ & $1.7 \cdot 10^{-5}$ \\
\hline 16 & 6.9 & $3.8 \cdot 10^{-12}$ & $3.2 \cdot 10^{-7}$ & $1.8 \cdot 10^{-11}$ & $1.5 \cdot 10^{-6}$ & $4.3 \cdot 10^{-11}$ & $4.5 \cdot 10^{-6}$
\end{tabular}

Table 6.2: Several smallest eigenvalues for 2D Laplacian.

Again, these results indicate the linear scaling of the tensor method in $n$. The cost of one power iteration step increases like $O(n \log n)$ as expected. Furthermore, the theory predicts the asymptotic behaviour

$$
\delta_{\lambda}=O\left(\lambda h^{2}\right) \quad \text { and } \quad \delta_{u}=O(\sqrt{\lambda} h), \quad \text { as } \quad h \rightarrow 0,
$$

which is in agreement with the above presented calculations. Notice that in our case the true scaling factor between the errors on sequential refined grids is 16 for the eigenvalues and 4 for the eigenfunctions.

Example 3. We apply the tensor method to compute the minimal eigenvalue of the 3D Laplacian on large $n \times n \times n$ grids with $n=2^{p}-1$ (see Table 6.1). In this case, the action of the truncation operator $T_{1}$ is equivalent to the rank-1 nonlinear Tucker approximation of the $n \times n \times n$, rank- $R$ tensors arising at each iterative step.

We observe the asymptotic complexity $O(d n \log n)$ to achieve the theoretical error bounds for both the eigenvalues and eigenfunctions. Notice that the problem size on the finest grid (with $n=2^{17}, d=3$ ) exceeds $N=n^{d}=2^{17 d} \approx 10^{15}$, which is far beyond the facilities of modern super-computers. Hence tensor methods are mandatory for solving large scale multi-dimensional spectral problems. 


\begin{tabular}{c|c|c|c|c}
$p$ & Time $/$ it. & $\delta_{\lambda}$ & $\delta_{u}$ & it. \\
\hline 6 & 0.03 & $2.0 \cdot 10^{-4}$ & $1.5 \cdot 10^{-3}$ & 4 \\
\hline 8 & 0.05 & $1.6 \cdot 10^{-5}$ & $9.4 \cdot 10^{-4}$ & 4 \\
\hline 10 & 0.12 & $7.8 \cdot 10^{-7}$ & $1.2 \cdot 10^{-4}$ & 5 \\
\hline 12 & 0.51 & $4.9 \cdot 10^{-8}$ & $3.4 \cdot 10^{-5}$ & 5 \\
\hline 14 & 2.2 & $3.1 \cdot 10^{-9}$ & $9.3 \cdot 10^{-6}$ & 5 \\
\hline 16 & 10.6 & $1.9 \cdot 10^{-10}$ & $2.8 \cdot 10^{-6}$ & 5 \\
\hline 17 & 22.3 & $4.8 \cdot 10^{-11}$ & $1.6 \cdot 10^{-6}$ & 5
\end{tabular}

Table 6.3: Minimal eigenvalue for 3D Laplacian on large spatial grids.

\subsection{Operator with variable coefficients}

In this section we consider eigenvalue problems for the elliptic operators of the form

$$
\Lambda u=\Delta u+c(x) u, \quad x \in \mathbb{R}^{d}
$$

with smooth coefficient $c(x)$.

In the subsequent examples we consider discretisations represented by matrices of the form

$$
\mathcal{A}=\mathcal{M}+q \mathcal{C}, \quad q>0,
$$

where $\mathcal{M}>0$ is the finite difference negative Laplacian in $2 \mathrm{D}$ as before, and $\mathcal{C}$ is the low Kronecker rank matrix discretising the zero-order term $c(x)=\sum_{k=1}^{R} c_{k}^{(1)}\left(x_{1}\right) \cdot c_{k}^{(2)}\left(x_{2}\right) \geq 0$ as in (4.1). We suppose that

$$
0 \leq\langle\mathcal{C} x, x\rangle \leq\langle x, x\rangle \quad \forall x \in \mathbb{V}_{n} \backslash\{0\}
$$

Example 4. In this example we apply the Lanczos algorithm for computing the largest eigenvalues. Consider matrices of the form

$$
\mathcal{A}=\mathcal{M}+\sum_{t=1}^{R} D_{t} \otimes D_{t}
$$

where $\mathcal{M}$ is the negative discrete Laplacian and $D_{t}$ are diagonal matrices with positive entries. We approximate the maximal eigenvalue by the standard Lanczos and truncated tensor Lanczos methods for the following two examples:

(A) the entries of $D_{t}$ are grid values of the function $\left(1+T_{t}(x)\right) / 10$, where $T_{t}$ is the Chebyshev polynomial of degree $t$;

(B) the entries of $D_{t}$ are random values uniformly distributed in $[0,1]$.

We compare the results obtained after 50 iterations for both methods. The matrix size is $N=300^{2}$, the truncation rank and the accuracy are set to 10 and $10^{-2}$, respectively. 


\begin{tabular}{c|c|c|c|c|c}
$R$ & 1 & 3 & 5 & 7 & 9 \\
\hline Standard Lanczos & 7.989 & 7.957 & 7.925 & 7.900 & 7.893 \\
\hline Tensor Lanczos & 7.977 & 7.940 & 7.917 & 7.893 & 7.906
\end{tabular}

Table 6.4: Maximal eigenvalues, case (A).

\begin{tabular}{c|c|c|c|c|c}
$R$ & 1 & 3 & 5 & 7 & 9 \\
\hline Standard Lanczos & 7.862 & 7.615 & 7.302 & 6.800 & 6.460 \\
\hline Tensor Lanczos & 7.852 & 7.608 & 7.292 & 6.789 & 6.452
\end{tabular}

Table 6.5: Maximal eigenvalues, case (B).

We observe that the computed eigenvalues in both methods are close to the truncation accuracy even for the random case. This suggests that tensor tools may be applied to a much broader class of matrices than required by the theory described above.

Example 5. The minimal eigenvalue is calculated for the problem (6.2) with the matrix $\mathcal{C}$ corresponding to the rank-1 potential $c(x)=\sin \left(\lambda x_{1}\right) \sin \left(\lambda x_{2}\right)$ in $(0,1)^{2}$. We perform the truncated power iteration with the matrix $\mathcal{L}=\mathcal{A}^{-1}$, such that the respective matrix-vector multiplication $y=\mathcal{L} U$ is implemented by the "truncated" iterative procedure, $y^{p} \rightarrow y$, as $p \rightarrow \infty$, where

$$
y^{p+1}=y^{p}-\omega \mathcal{L}_{R}\left(\mathcal{A} y^{p}-U\right), \quad p=0,1, \ldots
$$

with certain rank- $r$ initial guess $y^{0}$ and with a proper relaxation parameter $\omega \in(0,2)$. Here $\mathcal{L}_{R}$ is the rank- $R$ approximation to the inverse of the shifted Laplacian described in (6.1).

Table 6.6 presents the results on the sequence of $n \times n$ grids, $n=2^{p}, p=10,11,12,13$, for the truncation rank $r=3$. We give the total CPU time (sec.), the number of power iterations and the scaling factor between the neighbouring grids. We expect an $O(n \log n)$ time scaling provided that all nested iterations require the same number of loops and the same tensor rank for the preconditioner (of course, there are some fluctuations).

\begin{tabular}{c|c|c|c|c}
$n$ & Time & $\delta_{\text {Res }}$ & Power iter. & Scaling \\
\hline 1024 & 7.3 & $3.0 \cdot 10^{-4}$ & 10 & - \\
\hline 2048 & 23.6 & $1.5 \cdot 10^{-4}$ & 14 & 2.3 \\
\hline 4096 & 63.9 & $7.6 \cdot 10^{-5}$ & 14 & 2.7 \\
\hline 8192 & 209. & $3.8 \cdot 10^{-5}$ & 17 & 2.7
\end{tabular}

Table 6.6: Minimal eigenvalue for $-\Delta+c(x)$ in 2D.

This table indicates the linear-logarithmic scaling in $n$ as well as the robust convergence of the power iteration with the tensor modification.

\subsection{Concluding remarks}

The theoretical and numerical analysis of multi-dimensional eigenvalue problems presented in the paper clearly indicate that tensor structured methods for the approximation and 
solution of "smooth" spectral problems in $\mathbb{R}^{d}$ yield a promising basis for efficient solution methods in the modern high dimensional applications. Moreover, it seem that these methods are not restricted to smooth problems.

Acknowledgement. The authors are thankful to Dipl. Ing. Cristóbal Bertoglio (ParisVI) for assistence with the numerical computations of Tables 6.1, 6.2.

\section{References}

[1] I. Babuška and J. Osborn. Eigenvalue Problems. In P. Ciarlet and J. Lions, editors, Handbook of Numerical Analysis, Vol. II: Finite Element Methods (Part 1), pages 641788. Elsevier Science Publishers, Amsterdam, 1991.

[2] S.N. Bernstein: Leçons sur les propriétés extrémales et la meilleure approximation des fonctions analytiques d'une variable réelle. Paris: Gauthier-Villars 1926

[3] G. Beylkin and M. M. Mohlenkamp: Numerical operator calculus in higher dimensions, Proc. Natl. Acad. Sci. USA, 99 (2002), 10246-10251.

[4] G. Beylkin and M. M. Mohlenkamp: Algorithms for numerical analysis in high dimensions, SIAM J. Sci. Comput., 26 (2005), 2133-2159.

[5] D. Braess and W. Hackbusch: Approximation of $1 / x$ by exponential sums in $[1, \infty)$. IMA J. Numer. Anal., 25 (2005) 685-697.

[6] Chinnamsetty, S. R., Espig, M., Khoromskij, B. N., Hackbusch, W,. and H.-J. Flad: Tensor product approximation with optimal rank in quantum chemistry, Journal of Chemical Physics, 127 (2007), no.8, art-no. 084110.

[7] L. De Lathauwer, B. De Moor, and J. Vandewalle, On the best rank-1 and rank$\left(R_{1}, \ldots, R_{N}\right)$ approximation of higher-order tensors. SIAM J. Matrix Anal. Appl., 21 (2000) 1324-1342.

[8] M. Espig: Approximation mit Elementartensorsummen. Doctoral thesis, Universität Leipzig, 2008.

[9] H.-J. Flad, B. Khoromskij, D. Savostianov, and E. Tyrtyshnikov: Verification of the cross $3 d$ algorithm on quantum chemistry data. Rus. J. Numer. Anal. and Math. Modelling, 4 (2008), 1-16.

[10] I.P. Gavrilyuk, W. Hackbusch, and B.N. Khoromskij: Tensor-product approximation to elliptic and parabolic solution operators in higher dimensions. Computing 74 (2005), 131-157.

[11] W. Hackbusch. Elliptic Differential Equations. Springer Verlag, 1992.

[12] W. Hackbusch and B.N. Khoromskij: Low-rank Kronecker product approximation to multi-dimensional nonlocal operators. Part I. Separable approximation of multi-variate functions. Computing 76 (2006), 177-202. 
[13] W. Hackbusch, B.N. Khoromskij, and E.E. Tyrtyshnikov: Hierarchical Kronecker tensor-product approximations. J. Numer. Math. 13 (2005), 119-156.

[14] W. Hackbusch, B.N. Khoromskij, and E.E. Tyrtyshnikov: Approximate iterations for structured matrices. Numer. Math. 109 (2008), 365-383.

[15] B. N. Khoromskij: Structured Rank- $\left(r_{1}, \ldots, r_{d}\right)$ Decomposition of Function-related Tensors in $\mathbb{R}^{d}$. Comp. Meth. in Applied Math. 6 (2006), 194-220.

[16] B. N. Khoromskij: On tensor approximation of Green iterations for Kohn-Sham equations. Comp. and Visualization in Sci., 11 (2008) 259-271.

[17] B.N. Khoromskij: Tensor-structured Preconditioners and Approximate Inverse of Elliptic Operators in $\mathbb{R}^{d}$. J. Constr. Approx., 2009. DOI: 10.1007/s00365-009-9068-9.

[18] B.N. Khoromskij and V. Khoromskaia: Multigrid tensor approximation of function related multi-dimensional arrays. SIAM J. on Sci. Comp., 31(4), 3002-3026 (2009).

[19] B.N. Khoromskij, V. Khoromskaia, and H.-J. Flad: Numerical Solution of the HartreeFock Equation in Multilevel Tensor-structured Format. Preprint 44/2009, MPI MIS Leipzig 2009 (submitted).

[20] A. Knyazev: New Estimates for Ritz Vectors. Math. Comp., 66:985-995, 1997.

[21] T. G. Kolda and B. W. Bader: Tensor decompositions and applications. Technical Report No SAND2007-6702, Sandia National Laboratories, Albuquerque, NM and Livermore, CA, November 2007.

[22] J.M. Melenk: hp-finite element methods for singular perturbations. Springer Lecture Notes in Mathematics, 1796, (2002).

[23] M. Melenk and S. Sauter: Convergence analysis for finite element discretizations of the Helmholtz equation. Part I: The full space problem. 09-2008, Universität Zürich 2008.

[24] I.V. Oseledets, D.V. Savostyanov, and E.E. Tyrtyshnikov: Tucker dimensionality reduction of three-dimensional arrays in linear time. SIMAX, vol. 30, no. 3, pp. 939-956 (2008).

[25] E. Ovtchinnikov: Cluster robust error estimates for the Raleigh-Ritz approximation I: Estimates for invariant subspaces. LAA, 415(1):167-187, 2006.

[26] E.E. Tyrtyshnikov: Tensor approximations of matrices generated by asymptotically smooth functions. Sbornik: Mathematics 194, No. 5-6 (2003), 941-954 (translated from Mat. Sb. 194, No. 6 (2003), 146-160).

[27] E.E. Tyrtyshnikov: Kronecker-product approximations for some function-related matrices. Linear Algebra Appl. 379 (2004), 423-437.

[28] S. Sauter and C. Schwab: Randelementmethoden. B.G. Teubner, 2004.

[29] C. Schwab: p- and hp-Finite Element Methods. Oxford University Press, 1998. 
[30] A. Smilde, R. Bro, and P.Geladi: Multiway analysis. Wiley, 2004.

[31] E. Tadmor: The exponential accuracy of Fourier and Chebychev differencing methods. SIAM J. Numer. Anal. 23 (1986), 1-23.

[32] V. Thomée: Galerkin finite element methods for parabolic problems. Springer, Berlin, 1997.

[33] R.-A. Todor, and Ch. Schwab: Convergence rate for sparse approximation of elliptic problems with stochastic coefficients. IMA J. of Numer. Anal. 27 (2007), 232-261.

[34] L.R. Tucker: Some mathematical notes on three-mode factor analysis. Psychometrika 31 (1966) 279-311. 